\title{
Comparing diagnostic classification of neurobehavioral disorder associated with prenatal alcohol exposure with the Canadian fetal alcohol spectrum disorder guidelines: a cohort study
}

\author{
James L. Sanders PhD, Rebecca E. Hudson Breen PhD, Nicole Netelenbos MSc
}

Abstract

Background: Diagnostic criteria have recently been introduced in the Diagnostic and Statistical Manual of Mental Disorders, 5th edition (DSM-5), for neurobehavioral disorder associated with prenatal alcohol exposure (ND-PAE). The purpose of this study is to assess the classification of this condition using the Canadian fetal alcohol spectrum disorder (FASD) multidisciplinary diagnostic guidelines as the standard of comparison. First, classification of ND-PAE was compared with Canadian FASD diagnoses of fetal alcohol syndrome (FAS), partial FAS and alcohol-related neurodevelopmental disorder. Second, classification of ND-PAE was compared with FAS and pFAS only, a criterion for which includes facial features highly predictive of prenatal alcohol exposure and effects.

Methods: Eighty-two patients underwent multidisciplinary clinical evaluations using the Canadian FASD diagnostic guidelines between 2011 and 2015. Two clinicians independently reviewed patient files for evidence of diagnostic criteria for ND-PAE when applying an impairment cut-off level of 2 or more standard deviations below the mean, or clinically significant impairment in the absence of standardized norm-referenced measures.

Results: Good interrater reliability was established between clinicians $(\kappa=0.79)$. Classifications of ND-PAE and Canadian FASD diagnoses, including alcohol-related neurodevelopmental disorder, were moderately correlated (Cramer $V[82]=0.44, p<0.01)$. However, ND-PAE possessed low sensitivity in FASD identification. Further, there was no correlation between ND-PAE and FAS/pFAS classifications (Cramer V [82] $=0.05, p>0.05)$.

Interpretation: Although there is considerable overlap between both sets of criteria, ND-PAE was less likely to identify patients with FASD. Although the neurobehavioral domains assessed by ND-PAE are supported in research, its diagnostic structure restricts the identification of FASD at the impairment threshold of 2 or more standard deviations. A disconnect remains with regard to impairment thresholds between FASD, which relies on neurodevelopmental data, and ND-PAE, which relies on clinical judgment.

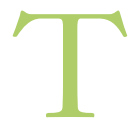

he effects of prenatal alcohol exposure on the developing central nervous system (CNS) are widespread, cutting across domains of intelligence, executive functioning, learning and memory, academic achievement, communication, visual-spatial ability, motor skills, attention and hyperactivity, externalizing behaviours and adaptive functioning. ${ }^{1}$ Early diagnosis is associated with improved long-term outcomes. ${ }^{2-4}$ Diagnostic criteria for fetal alcohol syndrome (FAS), including growth restriction, characteristic facial features and CNS dysfunction, were identified in early years of fetal alcohol research. ${ }^{5}$ It was soon apparent, however, that the CNS could be impacted by prenatal alcohol exposure in the absence of growth restriction and facial features. ${ }^{6}$ In subsequent years, diagnostic guidelines for fetal alcohol spectrum disorder (FASD) were developed. ${ }^{7-10}$ However, the diagnosis of FASD is not ubiquitous, with varying systems that lead to contradictory outcomes. ${ }^{11,12}$
Despite calls for consensus in FASD diagnosis, ${ }^{13}$ different multidisciplinary diagnostic systems continue to emerge..$^{14,15}$

In Canada, most FASD diagnostic settings have adopted the use of Canadian guidelines. ${ }^{16}$ In addition to confirming prenatal alcohol exposure, the Canadian guidelines identify 9 functional CNS domains to be examined in each patient. Substantial deficits of 2 standard deviations (SDs) or more below the mean, or clinically significant impairment in at least 3 areas of CNS criteria, are necessary for a diagnosis of FAS,

\section{Competing interests: None declared.}

This article has been peer reviewed.

Correspondence to: James Sanders, james.sanders@uleth.ca

CMAJ Open 2017. DOI:10.9778/cmajo.20160137 
partial FAS, or alcohol-related neurodevelopmental disorder. Diagnoses of FAS and partial FAS also include physical growth restriction and characteristic facial features. ${ }^{8}$ Recent revisions to these guidelines place greater emphasis on lifespan diagnosis and expansion of clinical domains. ${ }^{17}$

Current FASD diagnostic practices are multifaceted and complex, with the use of multidisciplinary teams being considered best practice. ${ }^{1}$ Although this approach results in comprehensive assessment, it is costly and limits clinical capacity. ${ }^{2}$ The development of more efficient diagnostic systems is needed to identify a wider range of patients affected by prenatal alcohol exposure and to improve access to services. ${ }^{18} \mathrm{With}$ the publication of the Diagnostic and Statistical Manual of Mental Disorders, 5th edition (DSM-5), ${ }^{19}$ criteria were developed for a prenatal alcohol exposure-related diagnosis: NDPAE. This system could serve as a more cost-effective and accessible approach in the diagnosis of FASD.

The purpose of this study is to compare the proportion of patients receiving a Canadian diagnosis of FAS, partial FAS or alcohol-related neurodevelopmental disorder to those meeting the criteria for ND-PAE. Although there is considerable overlap between the domains assessed in DSM-5 and those in the Canadian FASD guidelines, ${ }^{8}$ it is hypothesized that the DSM-5 criteria will identify fewer cases of ND-PAE. This is predicated on the DSM-5's organizational structure, which stipulates impairment across each of its 3 super-domains of neurocognitive functioning, self-regulation and adaptive functioning. In contrast, the Canadian guidelines require substantial deficits in 3 of any of its 9 functional CNS domains. ${ }^{8}$

The structuring of the adaptive functioning domain in DSM-5 requires the presence of impairment on at least 2 symptoms, with the presentation of either communication deficit or impaired social communication and interaction as criteria. Comparison is therefore made between the DSM-5 criteria and the Canadian guidelines, the latter requiring impaired adaptive functioning within conceptual, social or practical domains, and the former including domains of motor skills, receptive/expressive communication, social communication and interaction, and daily living skills.

In addition, the proportion of cases meeting criteria for ND-PAE are compared with those with FAS or partial FAS, which diagnoses include characteristic facial features highly specific to prenatal alcohol exposure..$^{20}$ These facial characteristics include short palpebral fissures, smooth philtrum and thin upper lip, and were measured in consort with the Canadian guidelines ${ }^{8}$ and the 4-digit code. ${ }^{7}$ Given the specificity of these facial features to prenatal alcohol effects, classification of ND-PAE should be correlated to FAS and partial FAS.

\section{Methods}

\section{Setting}

Data were collected through a multidisciplinary FASD diagnostic clinic in Alberta using the 2005 Canadian guidelines. ${ }^{8}$ The clinic team included physicians, a psychologist, speech/ language pathologists and occupational therapists trained in FASD diagnostic assessment. Each clinician assessed their respective domains, with speech/language pathologists assessing the receptive and expressive communication domain, and occupational therapists assessing the sensory motor domain.

Tests administered and the corresponding CNS domains were as follows: sensory motor (Bruininks-Oseretsky Test of Motor Proficiency, 2nd edition); intelligence (Wechsler Scales); receptive and expressive communication (Clinical Evaluation of Language Fundamentals, 4th edition); academic achievement (Woodcock Johnson Tests of Achievement, 3rd edition); memory (Children's Auditory Verbal Learning Test, 2nd edition, or California Verbal Learning Test, 2nd edition; Rey Complex Figure Test); executive function (Delis-Kaplan Executive Functioning Scales and Behavioural Rating Inventory of Executive Functioning); attention deficit/hyperactivity (Conners-3; DSM criteria for attention deficit-hyperactivity disorder); and adaptive behaviour, social skills and social communication (Adaptive Behaviour Assessment System, 2nd edition). The brain structure domain was assessed by occipitofrontal circumference or when diagnostic imaging was available. Significant deficits ( $\geq 2$ SD) or clinically significant impairment in at least 3 domains is necessary for a diagnosis of FAS, partial FAS and alcohol-related neurodevelopmental disorder, and confirmation of prenatal alcohol exposure is necessary for partial FAS and alcohol-related neurodevelopmental disorder diagnoses. ${ }^{8}$

Child and adolescent clinics were held for children ages 7-17 years, and adult clinics for patients aged 18 years and older. Confirmation of alcohol exposure was obtained from direct maternal self-report or professional documentation such as hospital, social work or police records.

\section{Patients}

Patients were assessed for participation between 2011 and 2015, most of whom were residing in Southern Alberta during the time of data collection. Sixty-three children and adolescents (mean age 11.1 [SD 3.4] yr) and 19 adults (mean age 29.1 [SD 9.9] yr) were assessed. Consecutive sampling was used to obtain participants. Patients referred to the local FASD diagnostic clinic and who had undergone multidisciplinary assessment for the purpose of diagnosis were eligible to participate. Referrals were accepted from a range of sources, including physicians, schools, social workers, psychologists and self-referral.

\section{Design}

A database was developed to include diagnostic and outcome data retrieved from patient files. This information included functioning on each of the 9 domains described in the 2005 Canadian guidelines, ${ }^{8}$ as well as prenatal risk factors and growth and facial measurements.

Two clinicians independently reviewed patient files for evidence of meeting the diagnostic criteria for ND-PAE. Although the Canadian guidelines specify the degree of deficit necessary for a symptom count (typically $\geq 2$ SD below the mean), the DSM-5 criteria makes no such distinction for most symptoms apart from identification of "impairment." One exception is within the intellectual domain, where DSM-5 does specify an intelligence quotient of 70 or below ( $\geq 2$ SD below the mean) as 
necessary for symptom identification. In this study, symptoms were classified based on scores 2 or more SDs below the mean where norm-referenced data were available, in line with generally accepted levels of impairment in neuropsychological assessment, and to establish interrater agreement. This decision was also based on the stipulation of the Canadian guidelines: to differentiate between an impairment of true dysfunction caused by prenatal alcohol exposure and a subtle impairment that may not necessarily be a product of prenatal alcohol exposure, a cut-off of 2 or more SDs below the mean is recommended. ${ }^{8}$ However, other researchers have used more moderate cut-off levels in translating norm-referenced measurement into descriptive clinical impairment for DSM-5 criteria. ${ }^{21}$

Domains of significant deficit on clinical assessments were identified as impairments on equivalent DSM-5 symptoms in the areas of intelligence, executive functioning, learning, memory, attention deficit, impulse control, communication, motor skills and adaptive behaviour, including daily living skills and social communication and interaction. Impairment in mood or behavioural regulation were assessed by the presence of a diagnosed mood, anxiety, or relevant externalizing behaviour disorder. Impairment in visual-spatial reasoning was assessed by scores at the second percentile or lower on the copy trial of the Rey Complex Figure Test ${ }^{22}$ and other clinical information as available. Case files were reviewed for additional information.

\section{Analysis}

Classification accuracy of the DSM-5 criteria was assessed against the results of multidisciplinary assessments conducted in the clinic from 2011 to 2015 . Because symptom identification and diagnoses are dichotomous in nature, cross tabulations were used to describe the data and Cramer $V$ was used to assess the correlations between criteria. Percent agreement and $\kappa$ were used to assess interrater reliability. ${ }^{23}$

\section{Ethics approval}

Ethical approval for the project was obtained through the University of Lethbridge Human Subject Research Committee. Informed consent was obtained from patients or their legal guardians for the use of the clinical data for research purposes.

\section{Results}

\section{Patients}

Eighty-two patients (41 male, 41 female) between the ages of 7 and 47 years participated in the study. Seventy-nine patients had confirmed prenatal alcohol exposure. Of these, 73\% $(n=$ $60)$ received a diagnosis under the umbrella of FASD, including FAS $(n=1)$, partial FAS $(n=13)$ and alcohol-related neurodevelopmental disorder $(n=46)$. Of those receiving an FASD-related diagnosis, 43 were children and 17 were adults. Tests $\left(\chi^{2)}\right.$ to assess the frequency of diagnosis between children and adults were not statistically significant $(p=0.07)$, but trended toward a higher proportion of diagnoses for adults.

\section{Interrater reliability}

Two clinicians independently reviewed each case, identifying symptom criteria and diagnoses for ND-PAE. Good interrater reliability was established $(\kappa=0.79)^{24}$ based on $90 \%$ agreement between the 2 raters on ND-PAE classification and non-classification. Discordant cases $(n=8)$ represented a small portion of the sample and were discussed between the 2 clinicians to reach agreement.

\section{Classification of ND-PAE}

Dichotomous classifications using the Canadian criteria (FASD or not FASD) and DSM-5 criteria (ND-PAE or not ND-PAE) were moderately correlated (Cramer $V$ [82] $=0.44$, $p<0.01)$. Against the 2005 multidisciplinary Canadian FASD guidelines, total classification accuracy of the DSM-5 was $61 \%$. Of particular importance, all classification errors were false-negative $(n=32)$, indicating that 32 cases obtained nonclassification in DSM-5 in the presence of classification using the Canadian guidelines. For this reason, the DSM-5 possessed inflated specificity $(100 \%, 95 \%$ confidence interval [CI] $87.7 \%-100.0 \%$ ) but low sensitivity (47\%, 95\% CI $33.7 \%-60.0 \%)$. Furthermore, the DSM-5 and 2005 Canadian guidelines adaptive functioning criteria were highly correlated (Cramer $V[82]=0.71, p<0.01)$. Neurobehavioral disorder associated with prenatal alcohol exposure failed to reliably identify the presence of partial FAS or FAS (Table 1). In addition, there was no correlation between identification of

Table 1: Diagnostic outcome comparisons between the DSM-5 and the 2005 Canadian FASD guidelines

\begin{tabular}{|c|c|c|c|c|}
\hline & & \multicolumn{2}{|c|}{$\begin{array}{l}\text { ND-PAE diagnosis } \\
\text { from DSM-5, no. (\%) }\end{array}$} & \multirow[b]{2}{*}{ Total, no. (\% } \\
\hline & & No & Yes & \\
\hline \multirow{3}{*}{$\begin{array}{l}\text { FASD diagnosis from multidisciplinary } 2005 \\
\text { Canadian guidelines }\end{array}$} & No diagnosis & $22(100.0)$ & $0(0.0)$ & $22(100.0)$ \\
\hline & ARND & $22(47.8)$ & $24(52.2)$ & $46(100.0)$ \\
\hline & pFAS & $9(69.2)$ & $4(30.8)$ & $13(100.0)$ \\
\hline Total & & 54 (65.9) & $28(34.1)$ & $82(100.0)$ \\
\hline
\end{tabular}


ND-PAE and FAS/partial FAS classification (Cramer $V$ [82] = $0.05, p>0.05)$. The proportion of the sample with an outcome of 2 or more SDs below the mean in each ND-PAE symptom is described in Table 2 .

\section{Interpretation}

The DSM-5 criteria for ND-PAE were moderately correlated with multidisciplinary clinical assessment using the Canadian guidelines. The presence of some correlation is expected, given that both systems assess a relatively consistent range of symptoms across cognitive, regulatory and adaptive domains. Although there is considerable overlap between the areas assessed in DSM-5 and those assessed in the Canadian FASD guidelines, the DSM- 5 criteria for ND-PAE were less likely to identify patients who met the Canadian neurobehavioral criteria for FAS, partial FAS and alcohol-related neurodevelopmental disorder while using a threshold of 2 or more SDs on normreferenced measures. Of particular note, ND-PAE criteria failed to reliably identify the presence of partial FAS and FAS, which diagnoses incorporate cardinal facial features that are highly specific to the effects of prenatal alcohol exposure. ${ }^{20}$

Although the impairment cut-off for ND-PAE may be conservative in this study, the low sensitivity of the DSM-5 criteria is in part rooted in its organizational structure. The DSM-5 criteria as is imply a shared neurobehavioral profile among patients affected by ND-PAE. More specifically, the DSM-5 guidelines specify that patients with ND-PAE will have impairment in neurocognitive functioning, self-regulation and adaptive functioning categories. In contrast, the Canadian guidelines, ${ }^{8}$ as well as other FASD classification systems, ${ }^{7,9}$ do not specify in which domains patients with FASD will have impairment. In addition, the adaptive functioning domain is weighted more heavily in the DSM-5 guidelines, whereby patients with ND-PAE will present with at least 2 of 4 adaptive functioning symptoms. In other FASD guidelines, no symptom domain is given additional weighting over another. Identification within the adaptive functioning super-domain in the DSM-5 was highly correlated to assessment of adaptive behaviour within the Canadian guidelines. However, a high correlation between such domains would be anticipated. The DSM-5 adaptive functioning super-domain includes assessment of communication deficits and motor skills, which are considered separate domains in the Canadian guidelines.

The DSM-5 domains are based on evidence of a broad range of neurocognitive, self-regulatory and adaptive functioning deficits that can be observed as problems of everyday living such as in social, learning and work-related contexts. ${ }^{25,26}$ However, research has yet to delineate a specific neurobehavioral profile or core symptoms of FASD. ${ }^{1}$ Given the variable effects of dose, timing and pattern of alcohol use during pregnancy, ${ }^{27}$ in conjunction with varying stages of CNS development, the identification of a central neurobehavioral profile common to all patients with FASD is formidable.

Table 2: Proportions of patients with significant impairment* in neurobehavioural disorder associated with prenatal alcohol exposure areas of the DSM- 5 separated by Canadian fetal alcohol spectrum disorder diagnostic classification

\begin{tabular}{|c|c|c|c|c|}
\hline \multirow[b]{2}{*}{ DSM-5 areas of significant impairment } & \multicolumn{4}{|c|}{ No. (\%) } \\
\hline & No diagnosis & ARND & pFAS & FAS \\
\hline \multicolumn{5}{|l|}{ Neurocognitive } \\
\hline Global intelligence & $0(0.0)$ & $18(39.1)$ & $6(46.2)$ & $0(0.0)$ \\
\hline Executive function & $4(18.2)$ & $33(71.1)$ & $9(69.2)$ & $1(100.0)$ \\
\hline Learning & $5(22.7)$ & $37(80.4)$ & $10(76.9)$ & $0(0.0)$ \\
\hline Memory & $0(0.0)$ & $25(54.3)$ & $7(53.8)$ & $0(0.0)$ \\
\hline Visual-spatial reasoning & $0(0.0)$ & $19(41.3)$ & $6(46.2)$ & $0(0.0)$ \\
\hline \multicolumn{5}{|l|}{ Self-regulation } \\
\hline Mood or behavioural regulation & $6(27.3)$ & $22(47.8)$ & $6(46.2)$ & $0(0.0)$ \\
\hline Attention deficit & $14(63.6)$ & $32(69.6)$ & $9(69.2)$ & $1(100.0)$ \\
\hline Impulse control & $11(50.0)$ & $20(43.5)$ & $7(53.8)$ & $1(100.0)$ \\
\hline \multicolumn{5}{|l|}{ Adaptive functioning } \\
\hline Communication & $3(13.6)$ & $28(60.9)$ & $8(61.5)$ & $0(0.0)$ \\
\hline Social communication and interaction & $9(40.9)$ & $26(56.5)$ & $7(53.8)$ & $0(0.0)$ \\
\hline Daily living skills & 4 (18.2) & 24 (52.2) & $5(38.5)$ & $0(0.0)$ \\
\hline Motor skills & 4 (18.2) & $10(21.7)$ & $0(0.0)$ & $0(0.0)$ \\
\hline
\end{tabular}




\section{OPEN}

Since the third edition of the manual, DSM has adopted an approach based on descriptive, observable symptomology. ${ }^{28,29}$ In contrast, FASD diagnostic approaches use norm-referenced neurodevelopmental data. Although the domains assessed in ND-PAE and FASD diagnostic systems are largely harmonious, the impairment thresholds between them are unclear, with FASD systems using norm-based assessment, and ND-PAE using clinical judgment. However, several domains (i.e., learning, memory, intelligence quotient, executive functioning) are most appropriately assessed through neurodevelopmental assessment, while other domains (i.e., affect regulation, impulse control) may be most appropriately assessed through clinical description. In short, FASD diagnostic approaches over the past 15 years do not directly translate into DSM ideology of observable description. Nevertheless, the pursuit of more efficient approaches to FASD diagnosis such as ND-PAE is worthwhile given the prevalence and cost of FASD and limited clinical capacity leading to under-diagnosis in Canada. ${ }^{30}$

\section{Strengths and limitations}

The findings of this study were affected by 3 main aspects of the methodological design. First, although the Canadian guidelines were used as the reference standard, there are several guidelines in use around the world without an unequivocal gold standard. The outcomes could vary if one of the other guidelines had been selected. ${ }^{11}$ Second, the sensitivity and specificity analysis would have likely yielded different results if alternative cut-offs (e.g., $\geq 1.5$ or $\geq 1$ SD) were used in identifying significant impairments when applying the DSM-5 criteria. By lowering the cut-off level or omitting it all together, one would expect more patients with or without FASD to receive a classification as ND-PAE when using the DSM-5. Third, ND-PAE diagnoses were derived retroactively without the use of a clinical team, whereas the diagnostic outcomes from the Canadian guidelines were derived prospectively by a multidisciplinary team.

The use of a comprehensive multidisciplinary assessment is important, given that this approach considers additional variables such as external, developmental or familial factors independent of the criteria that may result in a diagnosis or non-diagnosis on a case-by-case basis. This type of evaluation is comprehensive and conducive to a more reliable diagnosis and recommendation for services for those affected by FASD. External, developmental or familial factors could not be fully accounted for in applying DSM-5 criteria retroactively to case files.

Importantly, accuracy of classification based on DSM-5 guidelines in this study is limited by lack of the absence of a case definition for what constitutes impairment. Bearing this in mind, significant deficits were considered impairments at a cut-off of 2 or more SDs. By imposing a threshold level, strong interrater agreement between the 2 independent reviewers was established. However, future research could further explore these impairment thresholds when applying different cut-offs or when using interview or questionnaire data.

The timeliness of this study is important given the recent publication of the DSM-5 and the stated need to evaluate cri- teria for ND-PAE. ${ }^{18,26}$ The multidisciplinary clinical assessments allowed for in-depth evaluation of each patient, accounting for external and developmental factors. Although informed by the 2005 Canadian guidelines, clinical assessments were more comprehensive than the cursory classification of patients based on file data. Finally, this study provides an explanation that the low sensitivity of the DSM-5 criteria at this impairment threshold is affected by the structure of the 3 super-domains, not the symptoms themselves.

\section{Conclusion}

Although the neurobehavioral domains assessed by ND-PAE are supported in the research, its diagnostic structure could limit the identification of patients with FASD. Meanwhile, there remains a need to establish an agreed-upon impairment threshold for ND-PAE for effective implementation into clinical practice. Further, some domains are assessed primarily through norm-referenced testing, while others are assessed through clinical description. However, we recommend that all potential symptom criteria should be considered in assessment of FASD until core or central features are delineated in a shared neurobehavioral profile.

\section{References}

1. Mattson SN, Crocker N, Nguyen TT. Fetal alcohol spectrum disorders: neuropsychological and behavioural features. Neuropsychol Rev 2011;21:81-101.

2. Benz J, Rasmussen C, Andrew G. Diagnosing fetal alcohol spectrum disorder: history, challenges and future directions. Paediatr Child Health 2009;14:231-7.

3. Olson HC, Jirikowic T, Kartin D, et al. Responding to the challenge of early intervention for fetal alcohol spectrum disorders. Infants Young Child 2007; 20:172-89.

4. Streissguth AP, Bookstein FL, Barr HM, et al. Risk factors for adverse life outcomes in fetal alcohol syndrome and fetal alcohol effects. 7 Dev Behav Pediatr 2004;25:228-38.

5. Jones KL, Smith DW, Ulleland CN, et al. Pattern of malformation in offspring of chronic alcoholic mothers. Lancet 1973;1:1267-71.

6. Clarren SK, Smith DW. The fetal alcohol syndrome. N Engl f Med 1978; 298:1063-7.

7. Astley SJ, Clarren SK. Diagnosing the full spectrum of fetal alcohol-exposed individuals: introducing the 4-digit diagnostic code. Alcobol Alcobol 2000; 35:400-10.

8. Chudley AE, Conry J, Cook JL, et al.; Public Health Agency of Canada's National Advisory Committee on Fetal Alcohol Spectrum Disorder. Fetal alcohol spectrum disorder: Canadian guidelines for diagnosis. CMAf 2005;172(Suppl):S1-21.

9. Hoyme HE, May PA, Kalberg WO, et al. A practical clinical approach to diagnosis of fetal alcohol spectrum disorders: clarification of the 1996 institute of medicine criteria. Pediatrics 2005;115:39-47.

10. Stratton K, Howe C, Ballaglia F, editors. Fetal alcohol syndrome: diagnosis, epidemiology, prevention, and treatment. Washington (DC): National Academy Press; 1996.

11. Coles CD, Gailey AR, Mulle JG, et al. A comparison among 5 methods for the clinical diagnosis of fetal alcohol spectrum disorders. Alcohol Clin Exp Res 2016; 40:1000-9.

12. Astley SJ. Comparison of the 4-digit diagnostic code and the Hoyme diagnostic guidelines for fetal alcohol spectrum disorders. Pediatrics 2006; 118:1532-45.

13. Astley SJ. Invited commentary on Australian fetal alcohol spectrum disorder diagnostic guidelines. BMC Pediatr 2014;14:85.

14. Landgraf MN, Nothacker M, Heinen F. Diagnosis of fetal alcohol syndrome (FAS): German guideline version 2013. Eur 7 Paediatr Neurol 2013;17: 437-46.

15. Watkins RE, Elliott EJ, Wilkins A, et al. Recommendations from a consensus development workshop on the diagnosis of fetal alcohol spectrum disorders in Australia. BMC Pediatr 2013;13:156.

16. Clarren SK, Lutke J, Sherbuck M. The Canadian guidelines and the interdisciplinary clinical capacity of Canada to diagnose fetal alcohol spectrum disorder. 7 Popul Ther Clin Pharmacol 2011;18:e494-9.

17. Cook JL, Green CR, Lilley CM, et al.; Canada Fetal Alcohol Spectrum Disorder Research Network. Fetal alcohol spectrum disorder: a guideline for diagnosis across the lifespan. CMAJ 2016;188:191-7. 
18. Olson HC. Advancing recognition of fetal alcohol spectrum disorders: the proposed DSM-5 diagnosis of "Neurobehavioral Disorder Associated with Prenatal Alcohol Exposure (ND-PAE).". Curr Dev Disord Rep 2015;2:187-98.

19. Diagnostic and Statistical Manual of Mental Disorders (DSM-5). 5 th ed. Washington (DC): American Psychiatric Association (APA); 2013.

20. Suttie M, Foroud T, Wetherill L, et al. Facial dysmorphism across the fetal alcohol spectrum. Pediatrics 2013;131:e779-88.

21. Rich S. Neurodevelopmental disorder associated with prenatal alcohol exposure: diagnosis and treatment of adolescents and adults in an era of DSM-5. Proceedings of the Seventh National Biennial Conference on Adolescents and Adults with Fetal Alcohol Spectrum Disorder (FASD); 2016 Apr. 6-9; Vancouver. Ottawa: Canadian Association of Schools of Nursing.

22. Meyers J, Meyers K. Rey complex figure test and recognition trial. Odessa (FL): Psychological Assessment Resources; 1995.

23. Cohen J. A coefficient of agreement for nominal scales. Educ Psychol Meas 1960;20:37-46.

24. McHugh ML. Interrater reliability: the kappa statistic. Biochem Med (Zagreb) 2012;22:276-82

25. Doyle LR, Mattson SN. Neurobehavioral Disorder Associated with Prenatal Alcohol Exposure (ND-PAE): review of evidence and guidelines for assessment. Curr Dev Disord Rep 2015;2:175-86.

26. Kable JA, O'Connor MJ, Olson HC, et al. Neurobehavioral Disorder Associated with Prenatal Alcohol Exposure (ND-PAE): proposed DSM-5 diagnosis. Child Psychiatry Hum Dev 2016;47:335-46.

27. O'Leary CM, Nassar N, Zubrick SR, et al. Evidence of a complex association between dose, pattern and timing of prenatal alcohol exposure and child behaviour problems. Addiction 2010;105:74-86.
28. Jablensky A. Living in a Kraepelinian world: Kraepelin's impact on modern psychiatry. Hist Psychiatry 2007;18:381-8.

29. Sanders JL. A distinct language and a historic pendulum: the evolution of the Diagnostic and Statistical Manual of Mental Disorders. Arch Psychiatr Nurs 2011;25:394-403.

30. Popova S, Lange S, Burd L, et al. Cost of fetal alcohol spectrum disorder diagnosis in Canada. PLoS One 2013;8:e60434.

Affiliation: Faculties of Health Sciences (Sanders, Hudson Breen) and Arts \& Science (Netelenbos), University of Lethbridge, Lethbridge, Alta.

Contributors: James Sanders contributed to the conception and design, data collection and analysis, interpretation, and drafting and revising of the manuscript. Rebecca Hudson Breen contributed to the data analysis and interpretation, and drafting of the manuscript. Nicole Netelenbos contributed to the data collection, and drafting and revising the manuscript. All of the authors approved the final version for publication and agreed to act as guarantors of the work.

Acknowledgements: The authors thank the Lethbridge FASD Diagnostic Clinic for their assistance and partnership in this study.

Supplemental information: For reviewer comments and the original submission of this manuscript, please see www.cmajopen.ca/content/5/1/ E178/suppl/DC1 